\title{
Millimeter per Two Hours
}

National Cancer Institute

\section{Source}

National Cancer Institute. Millimeter per Two Hours. NCI Thesaurus. Code C105509.

A unit of both speed (scalar) and velocity (vector), defined as the distance of one

centimeter traveled per unit time equal to two hours. 